\title{
Psicopatología del niño en la era del custo a la cifra: antecedentes, paradojas y posibles respuestas
}

\section{Child psychopathology in an era fascinated by numbers: background, paradoxes and posible answers}

Héctor García de Frutos*1

El artículo analiza la forma de clasificación actual de los malestares de la infancia y la adolescencia. Localiza los cimientos de la infancia en el nacimiento de la industria y la sistematización de la escuela, y de las taxonomías contemporáneas en el viraje epistémico que supuso el DSM III. Expone algunas inconsistencias de este modelo, y aboga por la clínica psicoanalítica: permite una aproximación distinta y consecuente a la psicopatología por autorizar la elucidación del paciente.

Palabras clave: DSM, psicopatología infanto-juvenil, psicoanálisis, genealogía, ideología

*1 Universidad de Barcelona (Barcelona, Espanha). 


\section{ARTIGOS}

\section{Introducción: clínica sin actos o explicaciones}

Se admite que el giro epistemológico del DSM III ha marcado el devenir de la psicopatología contemporánea. La psicopatología clínica, articulada en torno de las nociones de síntoma y síndrome, cedió en favor de la psicopatología estadística, cimentada en el criterio conductual y el constructo de trastorno. El DSM III no fue solo un manual prometedor ante la comercialización a gran escala de los psicofármacos en la década de los 70 en los EEUU. Tampoco únicamente la réplica del cientificismo ${ }^{1}$ al clamor suscitado por el ya casi olvidado movimiento antipsiquiátrico. Ni exclusivamente la materialización del anhelo de devolverle a la psiquiatría su aura científica mediante el uso de la cifra, en plena hegemonía del modelo biomédico en medicina. En EEUU el DSM III se erigió en respuesta administrativa eficaz a la creciente demanda de atención al padecimiento psíquico, influyendo decisivamente en Europa en la misma dirección.

Sin embargo, el empuje a la gestión eficaz de la salud mental pública no es nuevo: las clasificaciones públicas como la sección $\mathrm{F}$ de trastornos mentales de la CIE (incluida por primera vez en la CIE 6 en 1949) o el DSM (cuyo primer borrador, el Medical 203, se gestó en el ámbito de la psiquiatría militar, de la mano de William C. Menninger, tras la Segunda Guerra Mundial) nacen y persisten con vocación de gestionar poblaciones. Se constituyen pues tras el horror del

${ }^{1}$ Ideología basada en un autoritarismo científico que postula que cualquier problemática social debe resolverse con metodología inspirada en la ciencia, que ignora decididamente todos los límites del saber que las ciencias ha puesto de manifiesto (Peteiro, 2010), y que tiene en la promesa de progreso constante e ilimitado su dogma de base. 
exterminio sistematizado, y en los albores de la cibernética: se constataba que la razón humana producía monstruos, y que era posible una razón artificial sin falla, aséptica. ¿En qué supuso el DSM III un "avance” en el empuje biopolítico? En que inauguró la Evidence Based Medicine en el campo de los trastornos mentales. Ello, con la connivencia de la promoción del modelo biopsicosocial en salud mental (Engel, 1977), contemporáneo al nuevo DSM.

Hoy, la clasificación orientada a lo público apunta esencialmente a "categorizar datos" con el fin de homogeneizar el lenguaje de los agentes implicados (Gobierno, investigadores y médicos), y decidir "contrataciones de servicios", "discapacidades" o "intervenciones" (Sartorius, 2012). El dato y la categoría de la que resulta (no existen los datos brutos, pero mucho menos en el ámbito psicopatológico) determinan así las prácticas que convienen a la salud pública. En el horizonte: la salud mental como imperativo del desarrollo humano (Püras, 2017). Pero concebir que la práctica taxonómica consista esencialmente en categorizar datos conlleva lógicamente una devaluación decisiva del factor explicativo. A la par, este formateado elide el acto clínico en favor del protocolo, y facilita el recurso al test psicométrico.

Esta doble determinación de exclusión (del factor explicativo y del 40 acto clínico) incide particularmente en la clínica con niños y adolescentes: se torna iterativa, carente de matices, ortopédica. En el diagnóstico, tiende a desentenderse de la historia subjetiva, reduce al mínimo la anamnesis, banaliza la palabra del niño y opta esencialmente por medir sus capacidades. El uso sistemático de tests psicométricos excluye definitivamente la sorpresa: toda respuesta no medible es descartada. En el tratamiento, es afín al uso de medicación, a la indicación de pautas generalizadas, así como a la prescripción de tratamientos reeducativos o de inspiración conductual. Nuestra primera hipótesis de trabajo es que esta orientación tiene precedentes.

La institucionalización de la infancia desde el siglo XVIII

Sólo es posible distinguir una psicopatología específicamente infantojuvenil si previamente se ha concebido la infancia como estado diferenciado del desarrollo. Si le debemos a Rousseau la distinción filosófica entre niño y adulto, el recurso a la historia permite razonar que la infancia como hoy se concibe en salud mental deriva de dos modificaciones sociales fundamentales: la escuela, y la salud pública. 


\section{ARTIGOS}

Es decisivo a este respecto el siglo XVIII. Es el de Rousseau, pero también el de la revolución industrial, que dio lugar a "un encasillamiento más tupido de la población" (Foucault, 1999). El pobre fue desacralizado (sabemos del valor moral fundamental de la pobreza para el cristianismo) y categorizado por el estadista: buenos y malos pobres, vagos voluntarios, parados involuntarios, aptos, incapaces... También el loco: la cristalización definitiva de la enfermedad nerviosa se produjo durante este siglo a raíz de la publicación de numerosas monografías de autores británicos y franceses (Álvarez, Esteban y Sauvagnat, 2004). Era preciso elevar el nivel de salud del conjunto del cuerpo social, e incorporar al máximo número posible de sujetos al aparato productivo industrial. Es decir: preservar, mantener e incrementar la fuerza de trabajo. Ello establece las bases para la consolidación del higienismo en el siglo XIX. Los niños de clases populares son incorporados a la mano de obra industrial, y eso reconfigura las políticas de natalidad, supervivencia infantil y desarrollo óptimo de la población. Aunque el hijo sigue sumiso al orden patriarcal, el Estado se encargará de promover entre los padres obligaciones sobre el cuidado físico, la lactancia, la vestimenta y el ejercicio. La familia ya no es solamente un sistema de parentesco, un estatuto social, un sistema de transmisión de bienes; es un medio físico denso, que envuelve y mantiene el cuerpo del niño (Foucault, 1999). En definitiva, la modernidad es la época en que nace la inquietud por el niño en su calidad de cuerpo-infantil; "la invención de la infancia se produce y concreta en el contexto de una «familiarización» creciente de la sociedad que se despliega desde el siglo XVIII y donde la medicina tiene un papel protagonista" (Orellana, 2005). Constatamos como la conformación de la infancia depende entonces de políticas públicas y del discurso hegemónico, que cincelan un cambio en las formas de vida promovidas por las mutaciones en la economía productiva y la mano de obra. Puede quizás establecerse un paralelismo con el siglo XXI: hoy la salud mental de la infancia, la garantía de la plenitud en sus capacidades cognitivas, la promesa de su autonomía, creatividad y resiliencia, son requisitos que responden a la reconfiguración del mercado laboral que han producido la revolución de la información, el declive de la economía real y la robotización creciente.

La construcción de un modelo de niñez, acorde con una política de normalización social, depende en cualquier caso de la institución escolar (Orellana, 2005). Es comprometido fechar el origen de la institución escolar; situemos pues su lógica discursiva: es la creencia en que la formación libre de los niños dentro del medio adulto y familiar debe sustituirse por la delegación de la labor educativa a especialistas en una institución cerrada que 
aísla los cuerpos infantiles. Se institucionaliza la fisura entre el saber escolar y el de la vida cotidiana: la separación escolar hace de la escolarización un proceso materialmente separado del proceso de producción (Laporte, 1975). Fábrica y escuela son espacios cerrados y reglamentados. El alumno y el obrero encarnan el lugar del no saber: todo el saber del que pudieran disponer resultaría inútil y será desacreditado. Se establecen en la escuela técnicas de reglamentación y orden de la vida diaria; los contenidos a enseñar se jerarquizan y universalizan. El ejemplo paradigmático sea quizás el modelo establecido por Juan Bautista La Salle en el siglo XVIII: el que ve la consagración del "Santo Pedagogo", fundador de los hermanos de las Escuelas Cristianas y hoy patrono de los educadores. El furor educandis que Grimm detectó como la pasión de su siglo lo anima. La disciplina universal sobre los cuerpos de los hijos de las clases populares (las escuelas eran gratuitas) es aplicada por Hermanos, modelos a imitar, sustitutos de unos padres que "han abandonado a los hijos a sus propias conductas". La Regla, sostenida en la mirada omnipresente de Dios, se impone en el vestir, la limpieza, el mirar y el decir: es imperativo que el cuerpo del niño no sea sede de goce (Grosrichard, 1975).

Si en los siglos XVIII y XIX se impuso el higienismo con la aparición de la revolución industrial, la época contemporánea vive en el primer mundo la consolidación de la salud mental como preocupación social (¿laica?) de primer orden. La competitividad inter pares, particularmente en el ámbito del predominante sector servicios, formatea tecnologías del yo afines a los nuevos mercados. El imperativo social demanda emprendedores y personalidades hechas a sí mismas. El reverso se presenta en la escuela: el discurso cientificista promueve el cribado universal para clasificar a los niños en función de sus competencias e identificar los sujetos con trastornos para proponerles tratamientos específicos de su déficit. Establece su primacía degradando los saberes pedagógico y educativo: éstos carecen de aval científico y eficacia empíricamente demostrada, son impotentes para abordar a los chicos con trastorno, culpabilizan al niño al desconocer su afectación deficitaria real (al nivel del cerebro). Pero también devalúa el saber intuitivo de los progenitores acerca de las particularidades del hijo, reconfigurando la filiación: los padres no son responsables de los síntomas de sus hijos, pero la genética transmitida es un factor de riesgo decisivo. Encontramos en La Salle un precedente de la voluntad de negación del cuerpo como espacio de satisfacciones, hoy retomada por el cientificismo que reduce el cuerpo al organismo, y el síntoma a la disfunción cerebral. 


\section{ARTIGOS}

La cifra sustituye al ideal en un nuevo higienismo; y lo propio del cifrado es producir una razón sin sentido, ahistórica e incorporal.

\section{Taxonomías de la cifra para sujetos sin pasado}

Si bien conviene elucidar el presente por la lectura del pasado, la lógica del paralelismo histórico corre el riesgo de pecar de "anacronismo historiográfico": el estudio del pasado manteniendo un ojo puesto en el presente, la escritura ahistórica de la historia en la medida en que extrae el dato fuera del contexto social y político (Huertas, 2001). La teoría de la información, que hace equivaler dato e idea, es propensa a este desliz. En la metáfora computacional que gobierna la psicología el dato se pretende apolítico. Una vez extraído de la persona objeto de estudio afirma una verdad unívoca, libre de las huellas reales de la cronología (aunque sea un dato fechado): la serie de medidas produce un destino aséptico. Eso no excluye sin embargo la comprensión y los prejuicios. En el procesamiento computarizado, el algoritmo aísla un contexto por vecindad semántica entre términos y el número de veces que aparecen juntos, instaurando un pensamiento hegemónico que excluye su fundamento: la autoridad ética o moral, la lectura fundada en el juicio, la enarbolación argumentativa. Es la clase de razón que opera tras los ítems del test, o los criterios diagnósticos del DSM: gobiernan no pese a, sino por ser reiterativos. Son lenguajes cerrados sobre sí que cortocircuitan la palabra dicha y la lengua. Los informes diagnósticos devienen textos sin autor cuya pretendida objetividad enmascara el silenciamiento de la historia del sujeto y su familia. Esta metodología obtiene el apoyo de no pocas progenitores, que prefieren no saber nada de eso que podría concernirles íntimamente. ¿Pero es sólido su fundamento epistémico?

Puede constatarse una doble tendencia en la psiquiatría mainstream, ejemplificada en Sartorius (2012): por un lado, una vez admitida la precariedad del conocimiento científico respecto de la patogénesis del trastorno mental, se avala el "lenguaje común" del proyecto DSM por otorgar a los clínicos una gran fiabilidad diagnóstica. Por el otro, se alerta de que la clasificación reconocida obstaculiza la investigación innovadora, mientras se desprecian las clasificaciones locales y se explicita el deseo de homogeneidad en los tratamientos. El DSM progresa a partir de las últimas y mejores investigaciones científicas, pero a la vez es desacreditado (como hizo Insel, 2013), 
director del NIMH, duas semanas antes de su publicación) por su nula validez científica: las categorías diagnósticas se validan finalmente por consenso.

Esta doble tendencia es complementaria: impulsa el desvío de fondos al revolucionario proyecto $\mathrm{RDoC}$, que busca marcadores biológicos y conductuales transclínicos (Reardon, 2017), mientras en la práctica clínica la gestión de poblaciones en base a un diagnóstico prefabricado prospera, alimentada por el "fetichismo de la cifra" (Laurent, 2013a): un goce que las autoridades sanitarias incentivan sin descanso. Se revela una posición ética heredera del cinismo: la fiabilidad es fruto de la decidida estrechez de exploración mediante criterios observacionales reiterativos; la misma lógica debe imperar en la evaluación y selección de los tratamientos, quedando el contradictorio pedido de innovación y heterogeneidad epistémica sin consecuencias. En cuanto al RDoC, en cierto modo una reedición del proyecto RDC que en 1972 precedió la creación del DSM III (Shorter, 2013), conviene para mantener el statu-quo: las revoluciones restablecen, en su circularidad, al Amo en su sitio (Lacan, 1969-70/1992). El profesional puede elegir una u otra posición, pero seguirá usando diagnósticos establecidos y obtenidos por checklist, desechará posiciones fenomenológicas e hipótesis explicativas, y tanto su posición 44 subjetiva como la del paciente, tantas veces admitidas, quedarán excluidas del procedimiento real y su evaluación. El imperativo de la medida, la cientificidad y el ideal de progreso persisten. El cuestionamiento ético (¿quiero el bien de mi paciente? ¿Por qué motivos? ¿Por qué medios?) queda a lo sumo delegado a los comités de ética. No en vano, se responde ante las burocracias sanitarias y los imperativos sociales, no ante los vericuetos del propio deseo y las respuestas subjetivas consecuentes. El deseo inconsciente sigue siendo, en este siglo XXI, el elemento rechazado en la psicopatología. El uniformismo social propio de esta burocratización del saber sobre el padecimiento deriva en "una impotencia cada vez mayor del hombre para alcanzar su propio deseo" (Lacan, 1960/2005). Y es que el deseo, fundado en la lengua materna, remite a la paradoja de una semántica privada.

\section{El deseo y el síntoma psicoanalítico eluden la taxonomía}

El deseo inconsciente es objeto del psicoanálisis. Aunque los síntomas sean su índice, y tengan una presentación típica, dos síntomas nunca tienen la misma estructura y significación. Esta concepción objeta a la lógica taxonómica del diagnóstico psicopatológico, de la misma forma que el caso en 


\section{ARTIGOS}

psicoanálisis (singular) siempre objeta a la teoría (con pretensión universal). Objeta entonces a las taxonomías segregativas tipo DSM, que crean grupos homogéneos de pacientes con la misma etiología, curso, duración y resultado, para distinguirlos de lo normal, cuya medida es la distribución estadística.

Paradójicamente, el psicoanálisis norteamericano, quizás por sustituir la indagación del deseo inconsciente por el refuerzo del yo, abanderó las dos primeras versiones del DSM, pero curiosamente también decantó el giro decisivo del DSM III. Las primeras contemplaban los síndromes como reacciones del psiquismo, postulando la causalidad psíquica y habilitando la dimensión explicativa: de ahí la afinidad. En cambio, en el DSM III podemos situar dos vías de influencia: la adversa, por el rechazo que suscitó en algunos adalides de la psiquiatría estadística que abandonaron su formación analítica (Spitzer y Frances, entre otros); y la activa, pues distintos institutos y sociedades del psicoanálisis estadounidense consiguieron hacer pasar algunas enmiendas a la configuración del DSM III (Shorter, 2013).

Ahora bien, la articulación del psicoanálisis freudiano a la psicopatología fue de otro orden: nunca recusó el diagnóstico diferencial, ni negó la herencia que recibió de la psiquiatría clásica, pero su fundamento clínico es lo que puede elaborar el paciente de su síntoma. Freud reforzó así la distinción entre psicosis y neurosis: modificó la presentación clínica de la histeria, abrió la lectura significante de la neurosis obsesiva, y precisó los modos de autoerotismo en la psicosis (a la par que desestimó su tratamiento por el psicoanálisis). Mantuvo una posición ética muy distinta a la implícita en el modelo de trastorno, precisamente por universalizar el síntoma y darle al sujeto la posibilidad de leerlo. Una posición afín a la que promueve, hoy, el Alto Comisionado de los Derechos Humanos de las Naciones Unidas, cuando reclama una nueva aproximación que incluya la atención y el apoyo individualizados, centrados en la palabra, en detrimento de un modelo biomédico que ha promovido una excesiva e ineficaz medicalización de las enfermedades mentales (Püras, 2017). "Déjenos hablar" es el slogan que propone: no es otro que el de la inauguración del 'momento Freud' en la historia de la psicopatología.

\section{Paradojas e incidencias de los "trastornos del neurodesarrollo"}

La raíz actual de los trastornos de la infancia y la adolescencia reposa en el modelo biomédico evocado en el punto anterior. El DSM 5 mantiene la objetivación del trastorno infantil a la par que abandona el ateoricismo que fue 
su divisa desde el DSM III al sustituir la categoría "Trastornos de inicio en la infancia y la adolescencia" por "Trastornos del neurodesarrollo". Si en el DSM IV los trastornos del desarrollo eran solo los de tipo autístico, en el 5 los del neurodesarrollo subsumen el TDAH y los trastornos del aprendizaje, entre otros. Pero los trastornos catalogados bajo esta rúbrica carecen de biomarcadores probados. ¿Cómo puede un trastorno del neurodesarrollo carecer de biomarcadores? Muchos estudios pretenden aislar genes implicados en los trastornos mentales, o precisar su fundamento cerebral... pero son especulativos. No hay lesión, afectación o anomalía clínicamente observable. Y carecen de toda consecuencia diagnóstica o terapéutica aprobada: el diagnóstico sigue siendo clínico, y los tratamientos afines siguen anclados en fundamentos epistémicos anteriores a las consecuencias de la demostración experimental de la neuroplasticidad. Ésta lleva hoy a considerar lo neuronal y lo psíquico como campos causales diferenciados (Ansermet y Magistretti, 2006).

El cambio de nombre, por tanto, indica no solo la reintroducción de la teoría, sino cierta incoherencia en un manual cuya divisa es la eficacia administrativa y la utilidad de producir de un "lenguaje común". Se asevera un fundamento neural del trastorno; desconocemos los motivos, pero intenta46 remos elucidar algunos ejes de su andamiaje implícito.

Hay una primera paradoja que merece resaltarse: se postulan trastornos del neurodesarrollo, pero no hay episteme fundamentada sobre lo que sería un desarrollo neurotípico. Factores como la neuroplasticidad o la epigenética, por los que el organismo responde al ambiente, llevan más bien a postular la singularidad cerebral (Ansermet y Magistretti, 2006). La misma consideración subyace en la expresión 'neurodiversidad', que algunos sujetos con autismo defienden en los EEUU para despatologizar su ser.

El trastorno del neurodesarollo nos parece en cambio el correlato de la promoción de la noción de "espectro": en el autismo obviamente, pero de forma implícita en la lógica estadística propia de todo trastorno. Ya advertía Canguilhem (1971) que reducir la normalidad a su sola medida estadística conllevaba la anulación de la noción de enfermedad. En efecto, ¿dónde situar en el continuo de datos el punto de corte que distingue lo patológico de lo que no lo es? Un ejemplo: se mide cada vez más, pero son varios los trastornos del neurodesarrollo de los que ya no se especifica la prevalencia, cuando sí era explícita en el DSM IV.

Las nociones como "déficit" o "dificultad" son así epistémicamente frágiles. La única valoración de lo que no va es la medida cuantitativa: el trastorno carece de entidad propia, y por eso se define solo por algo que falta. 


\section{ARTIGOS}

Un trastorno es, per se, indefinible. La normalidad que necesita se estrecha hasta perder su esencia; pero no por intereses más o menos obscenos (Frances, 2014), que también, sino por la idiosincrasia misma del saber estadístico.

A la vez, como indica Allen, el DSM 5 hace más laxos algunos criterios diagnósticos y amplía su extensión. El TDAH, el TEA y el trastorno específico del aprendizaje (TA) presentan mayor prevalencia que en el DSM IV. Esto introduce una segunda paradoja: hay más niños diagnosticables, y tener un trastorno es casi cotidiano; pero a la vez la denominación de la nueva categoría reviste una semántica de mayor gravedad (el cerebro estaría realmente afectado).

¿Mayor casuística y declaración de causalidad biológica se traducen en más ventas de medicación? Sin duda, éste es uno de los grandes intríngulis del asunto. Digámoslo claro: el nuevo DSM 5 no va a acotar el crecimiento sostenido que vive la venta de metilfenidato para tratar el TDAH (ONU, 2012). Sin embargo, conviene recordar que si el viraje de los 80 hacia la psiquiatría científica ateórica de etiología biologicista fue impulsado por la comercialización de los psicofármacos, no se diseñó para ésta: son las farmacéuticas quienes adaptan sus productos al manual (Shorter, 2013). Además, hoy no hay psicofármacos eficaces para el tratamiento de los demás trastornos del neurodesarrollo. La operación DSM es más ideológica que comercial.

Un examen de la casuística permite constatar que este etiquetado cumple, socialmente, cierta función. Permite efectos de nominación en una infancia en la que padres y maestros presentan cada vez más dificultades para sostener su posición de autoridad; en que la estructura familiar se ha pluralizado; en que la vida es más líquida y global. La ciencia no solo universaliza, sino que produce términos que unifican. Cuando se usan siglas, algo muy común en los diagnósticos infantiles (TDAH, TEA, TA...), la pérdida de la semántica potencia la rigidez de la designación. Los sujetos pueden, así, hacer de su trastorno un nombre propio o un uso que les define y resignifica (Hacking, 1995).

En una época de decadencia del ideal, superado por la liberalización en los modos de vida, las producciones audiovisuales recuerdan que un trastorno puede ser desde una marca de estilo hasta una potencialidad excepcional. Uno puede, debe, ser su propio ideal. Así como el trastorno es deficiencia del propio cerebro, sin implicaciones psíquicas o familiares que lo contemplen como síntoma enlazado al Otro, se espera del menor que sea voluntarioso en combatir sus déficits; y más allá que elija, sea dueño de sí mismo, de su vida, de sus decisiones. Eso tiene su incidencia en los adultos. 


\section{Conclusiones: respuestas de la clínica psicoanalítica a la infancia generalizada}

En el primer apartado, situamos cierto paralelismo entre el obrero y el niño escolarizado: ambos son sujetos que al entrar en la institución son despojados de su saber propio para responder a un funcionamiento ajeno repetitivo. Cierta tipología de tratamiento de los trastornos mentales con niños y adolescentes se funda en la misma operación. Pero en el siglo XXI asistimos a otra clase de formateado del ser del hombre, el cual se revela abiertamente contradictorio: consiste en la promoción simultánea del hombre hecho a sí mismo, y del hombre sin atributos que se presta siempre a ser evaluado. Tienen algo en común: recusan las marcas de la propia historia.

Jacques Lacan acuñó el término de "niño generalizado" para pensar la subjetividad contemporánea, atrapada entre la captura del cuerpo por esta doble operación: su captura en el discurso de la ciencia, y su constitución como mercancía en el mercado global. El concepto común a estos cuerpos es el de goce puesto al servicio de un circuito. Si del cuerpo del obrero se extrae la fuerza de trabajo para producir una plusvalía distinta a sí mismo, el cuerpo del niño funciona en sí mismo como objeto de satisfacción para el adulto en posición materna. El niño generalizado es lo que aparece en la realidad de los hombres cuando, a diferencia del obrero, son a la vez cuerpo productor y mercancía producida. Es lo que la expresión "recursos humanos" hace resonar: el cuerpo mismo del hombre es el recurso. Es la forma en que proponemos leer la promoción del emprendedor, el self-made man, el freelance, indistintamente entre todas las clases sociales.

En cuanto al rechazo de la propia historia, remite a un tiempo de progreso no escandido. Da cuenta de ello la erosión marcada de la importancia de los ritos de paso en la sociedad capitalista contemporánea. Un rito de paso es un momento de ruptura subjetiva instituido culturalmente, marcado por el forzamiento de un significante distinto que viene a marcar una separación con uno anterior que signaba el ser del sujeto (Miller, 2014). Como mostró Freud, su modalidad princeps es el acto paterno de separación. El "niño generalizado" indica que los sujetos tienen hoy mayor dificultad para la separación. La modalidad de goce privilegia el lazo madre-hijo (Laurent, 2007). El consumismo, propio esencialmente de la pulsión oral, remite a esta no escansión en los tiempos del goce.

Es otro tiempo. Las instituciones no son hoy cerradas y homogéneas como la fábrica y la escuela de los siglos XVIII y XIX. La heterogeneidad de discursos 


\section{ARTIGOS}

es patente en la sociedad actual, siendo su devaluación manifiesta el signo distintivo de la época. Las palabras no sellan la creencia, y la cifra ha tomado ahí el testigo: en su vertiente contable (somos capitalistas de nosotros mismos), pero también en la mesurable. La fe propia de la clínica (lo creo si lo veo) ha dejado paso a la fe cientificista (lo creo si es medido). Los niños difícilmente pueden oponerse a ello, pero a muchos adultos hoy les resulta casi imposible.

A lo largo del texto hemos tratado de mostrar que el proyecto DSM, solidario de esta fe cientificista, se asienta en una serie de paradojas o aparentes contradicciones. Favorece cierta concepción de la psicopatología y la clínica con un marcado componente ideológico que encuentra sus antecedentes en modificaciones sociales y gubernamentales rastreables a partir del siglo XVIII. Esta ideología es la de hacer del signo un dato y del dato una cifra, y mediante la cifra instituir una categorización universal ajena al decir del paciente y el acto del profesional. Hemos defendido una salida alternativa: la concepción freudiana y lacaniana del síntoma. Si la civilización es un sistema simbólico que pretende la imposición del bienentendido, y la convergencia del deseo a partir de una homogeneización, la invención de Freud ha hecho posible que el sujeto pueda apropiarse de su historia inconsciente y producir otra cosa con ella. Para ello, como se sabe, la infancia es decisiva: es el momento más plástico a para que el niño pueda elaborar respuestas alternativas, pues las defensas no están aún establecidas (Miller, 2014). La rigidez de las terapéuticas reeducativas y cognitivo-conductuales lleva a la pérdida de una excelente oportunidad: la de que el sujeto pueda incidir, interpretación mediante, en sus marcas familiares.

Conviene una última aclaración: el psicoanálisis, por postular un uso posible del inconsciente freudiano, fundado en la represión y en la inercia de lo que quedó borrado, no puede ser equivalente a una psicopatología explicativa. Puede ser afín a una psiquiatría que dé valor a la anamnesis, al significado e historia de los síntomas, a su descripción detallista... pero no se confunde con ella. Ello, debido a que el auténtico diagnostico en psicoanálisis es siempre el autodiagnóstico (Miller, 1998), pues el saber es el del inconsciente que encamina al sujeto, no el del profesional. Y porque la propuesta del psicoanálisis (hacer existir el saber inconsciente mediante la hipótesis de un sujeto que lo articula con la verdad) no tiene pretensión universal. En eso, aporta ligereza a la práctica y permite una clínica del caso único, cada vez.

Sólo así, mediante la impronta de la diferencia que propone el "cada vez", es posible aislar una singularidad que no responda a las huellas indelebles de la propia existencia. Éstas, inconscientes, son algo más que una 
guía o una brújula para el sujeto: constituyen los raíles de un trayecto que no pocas veces acaba en la estación de destino prevista. Hablar, entonces, para desmontar parcialmente esos raíles, que son la norma a la que cada quién se halla ligado. Las variantes ideológicas a las que obedecemos son disfraces de ese destino pre-constituido. La cura analítica, entonces, si está guiada por el acto clínico que admita la sorpresa, permite al sujeto no sólo cierta subversión respecto de la norma ideal; sino también cierta transgresión natural del imperativo que lo hace homogéneo a su propia tendencia.

\section{Referencias}

Álvarez, J. M., Esteban, R., \& Sauvagnat, F. (2004). Fundamentos de psicopatologia psicoanalítica. Madrid, España: Síntesis.

American Psychiatric Association (2014). DSM-5, Manual diagnostico y estadístico de los trastornos mentales. Madrid, España: Médica Panamericana.

American Psychiatric Association (1987). DSM III R. Washington DC: APA.

American Psychiatric Association (1994). DSM IV. Washington DC: APA.

Ansermet, F., \& Magistretti, P. (2006). A cada cual su cerebro: plasticidad neuronal e inconsciente. Madrid, España: Katz.

Canguilhem, G. (1971). Lo normal y lo patológico. Buenos Aires, Argentina: Siglo XXI.

Engel, G. L. (1977, April). The need for a new medical model: a challenge for biomedicine. Science, New Series, 196(4286), 129-136.

Foucault, M. (1999). La política de la salud en el siglo XVIII. In Estrategias de poder (pp. 327-342). Barcelona, España: Paidós.

Frances, A. J. (2014). ¿Somos todos enfermos mentales? Barcelona, España: Ariel.

González Barrón, R. (Dir. y coord.) (2000). Psicopatología del niño y del adolescente. Madrid, España: Pirámide.

Grosrichard, A. (1975, mars). Le Saint Pédagogue. Ornicar 2, 19-40.

Hacking, I. (1995). The looping effects of human kinds. In D. Sperber, D. Premack, A. J. Premack, Causal cognition: a multidisciplinary debate (pp. 351-383). Cambridge, MA: Harvard University Press.

Huertas, R. (2001). Historia de la psiquiatría. ¿Por qué? ¿Para qué? Tradiciones historiográficas y nuevas tendencias. Frenia, I-1, 9-36.

Insel, Th. (2013). Transforming diagnosis. Thomas Insel's blog in the website of the National Institute of Mental Health, USA. Recuperado em 11ago.2018, de: 


\section{ARTIGOS}

$<$ https://www.nimh.nih.gov/about/directors/thomas-insel/blog/2013/transformingdiagnosis.shtml>.

Lacan, J. (1992). El seminario. Libro XVII, El reverso del psicoanálisis. Buenos Aires, Argentina: Paidós. (Trabajo original publicado en 1969-707).

Lacan, J. (2005). El triunfo de la religión, precedido del Discurso a los católicos. Buenos Aires, Argentina: Paidós. (Trabajo original publicado en 1960).

Lacan, J. (2012). Alocución sobre la psicosis del niño. In Otros Escritos. Buenos Aires, Argentina: Paidós. (Trabajo original publicado en 1967).

Laporte, D. (1975, mars). Histoire de l'éducation. Ornicar 2, $41-53$.

Laurent, É. (2007). Las nuevas inscripciones del sufrimiento en el niño. In S. Goldber, \& E. Stoisa, Psicoanálisis con niños y adolescentes: lo que aporta la enseñanza de Jacques Lacan. Buenos Aires, Argentina: Grama.

Laurent, É.(2013a). La batalla del autismo. Buenos Aires, Argentina: Grama.

Laurent, É. (2013b). La crisis post-DSM y el psicoanálisis 1 y 2. Látigo. Recuperado en 11 ago. 2008, de: <http://www.latigolacaniano.com/textos.html>. Acceso en: 11 ago. 2018.

Miller, J.-A. (1998). Elucidación de Lacan. Charlas Brasileñas. Buenos Aires, Argentina: EOL-Paidós.

Miller, J.-A. (2014). Prefacio. In H. Bonnaud. El inconsciente del niño. Barcelona, España: RBA/Gredos.

ONU, International Narcotics Control Board (2012). Annual reports. Recuperado en 11 ago. 2008, de: <http://www.incb.org/documents/Publications/AnnualReports/ AR2012/abuse_prescription_drugs.pdf) $>$.

Orellana, N. C. (2005). Foucault y el saber educativo. Segunda parte: La invención de la infancia. Revista electrónica Diálogos educativos, 5(9), 20-28.

Peteiro, J. (2010). El autoritarismo científico. Málaga, España: Miguel Gómez.

Püras, D. (2017). Special Rapporteur on the right of everyone to the enjoyment of the highest attainable standard of physical and mental health. Recuperado en 11 dic. 2008, de: <https://www.ohchr.org/EN/NewsEvents/Pages/DisplayNews. aspx? NewsID $=21480 \&$ LangID $=\mathrm{E}>$.

Reardon, S. (2017). US mental-health agency's push for basic research has slashed support for clinical trials. Recuperado en 10 sept. 2008, de:: <https://www.nature. $\mathrm{com} /$ news/us-mental-health-agency-s-push-for-basic-research-has-slashedsupport-for-clinical-trials-1.22145>.

Sartorius, N. (2012). Metaefectos de la clasificación de trastornos mentales. In D. A. Regier, W. E. Narrow, E. A. Kuhl, \& D. J. Kupfer, DSM-5: Evolución conceptual (pp. 55-74). Madrid, España: Panamericana.

Shorter, E. The history of DSM. In J. Paris, \& J. Phillips (Eds.) (2013). Making the 
DSM-5: Concepts and Controversies (pp. 3-20). New York, NY: Springer.

Whitaker, R. (2015). Anatomía de una epidemia. Madrid, España: Capitán Swing.

\section{Resumenes}

(Psicopatologia da criança na era do culto aos números: antecedentes, paradoxos e possíveis respostas)

$O$ artigo analisa a forma atual de classificação dos desconfortos da infância e adolescência. Localiza os fundamentos da infância no nascimento da indústria e na sistematização da escola e das taxonomias contemporâneas na virada epistêmica que supunha o DSM III. Expõe algumas inconsistências desse modelo e defende a clínica psicanalítica: permite uma abordagem diferente e consequente à psicopatologia para autorizar a elucidação do paciente.

Palabras-chave: DSM, psicopatologia infantil-juvenil, psicanálise, genealogia, ideologia

(Child psychopathology in an era fascinated byr numbers: background, paradoxes and possible answers)

This paper analyses the current form of classification of malaise in children and adolescents. It localizes the foundations of childhood in the birth of industry and the systemization of school, as well as those of contemporary taxonomies in the epistemic turn caused by the DSM III. It exposes some of the inconsistencies of this model and advocates for psychoanalytic cure, allowing a different and consequent approximation to psychopathology that allows to understand patients better.

Key words: DSM, childand adolescent psychopathology, psychoanalysis, genealogy, ideology

(Psychopathologie de l'enfant à l'ère du culte aux chiffres: antécédents, paradoxes et réponses possibles)

L'article analyse le mode de classification des malaises de l'enfance et de l'adolescence. Il localise les fondements de l'enfance dans la naissance de l'industrie et la systématisation de l'école et ceux des taxonomies contemporaines dans le virage épistémique propre au DSM III. Il expose quelques inconsistances de ce modèle et se range du côté de la clinique psychanalytique: il permet une approximation distincte et conséquente à la psychopathologie, ce qui permet de mieux comprendre les patients.

Mots clés: DSM, psychopathologie de l'enfant e de l'adolescent, psychanalyse, généalogie, idéologie 


\section{ARTIGOS}

(Psychopathologie bei kindern und Jugendlichen im heutigen rationellen Zeitalter: Vorgeschichte, Paradoxa und mögliche Antworten)

Dieser Artikel analysiert wie psychische Störungen von Kindern und Jugendlichen gegenwärtig eingestuft werden. Er zeigt auf, dass sich die Grundlagen der Kindheit in der Geburt der Industrie und der Systematisierung der Schule befinden und dass die zeitgenössischen Taxonomien das Resultat der epistemischen Wendung sind, welche vom DSM III verursacht wurde. Weiter werden Unstimmigkeiten dieses Modells aufgezeigt und die psychoanalytische Klinik als Alternative vorgeschlagen, da diese einen differenzierten und konsequenteren Zugang zur Psychopathologie ermöglicht, der es erlaubt, Patienten besser zu verstehen.

Schlüsselwörter: DSM, Psychopathologie bei Kindern und Jugendlichen, Psychoanalyse, Genealogie, Ideologie

Citação/Citation: Frutos, H. G. de (2019, março). Psicopatología del niño en la era del culto a la cifra: antecedentes, paradojas y posibles respuestas. Revista Latinoamericana de Psicopatologia Fundamental, 22(1), 38-53. http://dx.doi.org/10.1590/1415-4714.2018v22n1p38.3.

Editora da entrevista/Editor: Prof. Dr. Julio Verztman

Submetido/Submitted: 28.092018 / 09.28.2018 Revisado/Revised: 11.12 .2018 / 12.11.2018

Aceito/Accepted: 16.1.2019/1.16.2019

Copyright: (C) 2009 Associação Universitária de Pesquisa em Psicopatologia Fundamental/ University Association for Research in Fundamental Psychopathology. Este é um artigo de livre acesso, que permite uso irrestrito, distribuição e reprodução em qualquer meio, desde que o autor e a fonte sejam citados / This is an open-access article, which permits unrestricted use, distribution, and reproduction in any medium, provided the original authors and sources are credited.

Financiamento/Funding: Este trabalho não recebeu apoio / This work received no funding.

Conflito de interesses/Conflict of interest: Os autores declaram que não há conflito de

\section{Héctor García de Frutos}

Director del programa de máster y posgrado "Actuación clínica en psicoanalálisis y psicopatología"; Coordinador del programa de máster "Salud mental comunitária" ambos pela Universidad de Barcelona (Barcelona, España).

Passeig de la Vall d'Hebron 171

08035, Barcelona, España

https://orcid.org/0000-0001-5446-5354

hectorgarcia@ub.edu

This is an open-access article, which permits unrestricted use, distribution, (cc) BY-NC and reproduction in any medium for non-commercial purposes provided the original authors and sources are credited. 